\title{
Advantages of the flux-based interpretation of dependency length minimization
}

\author{
Sylvain Kahane \\ Chunxiao Yan \\ Modyco, Université Paris Nanterre \& CNRS \\ sylvain@kahane.fr \\ yanchunxiao@yahoo.fr
}

\begin{abstract}
Dependency length minimization (DLM, also called dependency distance minimization) is studied by many authors and identified as a property of natural languages. In this paper we show that DLM can be interpreted as the flux size minimization and study the advantages of such a view. First it allows us to understand why DLM is cognitively motivated and how it is related to the constraints on the processing of sentences. Second, it opens the door to the definition of a big range of variations of DLM, taking into account other characteristics of the flux such as nested constructions and projectivity.
\end{abstract}

\section{Introduction}

The dependency flux between two words in a sentence is the set of dependencies that link a word on the left with a word on the right (Kahane et al., 2017). The size of the flux in an inter-word position is the number of dependencies that cross this position. ${ }^{1}$ The flux size of a sentence is the sum of the sizes of the inter-word fluxes.

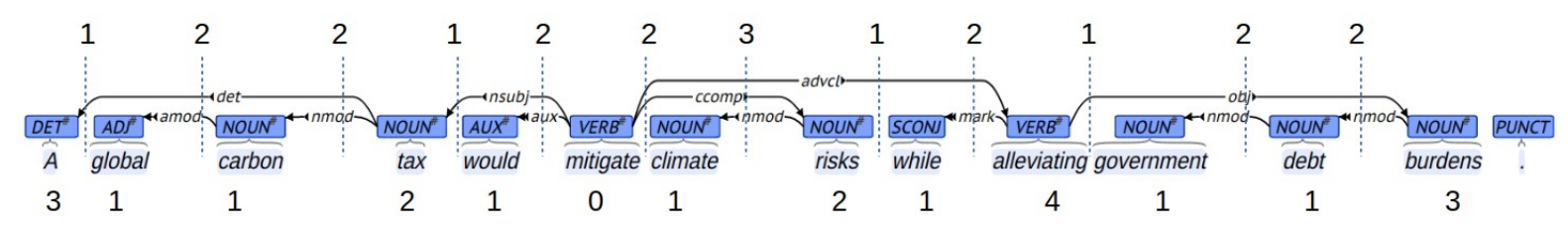

Figure 1. An ordered dependency tree with the flux size on top of each inter-word position and, under each word, the length of the dependency from its governor

On the top line, Figure 1 shows the size of the flux at each inter-word position. In the first position, between $A$ and global, there is only one dependency crossing $(A<\operatorname{det} \operatorname{tax})$; in the second position, between global and carbon, there are two dependencies ( $A<$ det tax; global $<$ amod carbon).

On the bottom line, Figure 1 shows, for each word, the length of the dependency that links that word to its governor. For example, the first word $A$ is linked to its governor tax by a dependency of length 3 because this dependency crosses 3 inter-word positions. The dependency length of a sentence is the sum of the lengths of the dependencies of that sentence.

It can be verified, for the sentence in Figure 1, that:

Dependency flux size of the sentence $=1+2+2+1+2+2+3+1+2+2+2+2=21$

Dependency length of the sentence $=3+1+1+2+1+0+1+2+1+2+1+4+1+1+3=21$

It is easy to check that the dependency length is always equal to the dependency flux size. Since the length of a dependency is the number of fluxes on which this dependency belongs, the size of the flux is

\footnotetext{
${ }^{1}$ The flux in a given position can also be viewed as the set of incomplete dependencies when only one of the two halves of the dependency structure is considered. Gibson (2000) introduces an Incomplete Dependency Hypothesis on the syntactic complexity, but he only considers dependencies of obligatory subcategorized elements.
} 
the sum, on all the dependencies, of the number of fluxes they cross. In other words, these two values are equal to the number of crossings between a dependency and an inter-word position.

Several studies have studied dependency length and shown that natural languages tend to minimize it (Liu, 2008; Futrell et al., 2015). This property is called dependency length minimization (DLM) or dependency distance minimization (dependency lengths can be interpreted as distances between syntactically related words). DLM is correlated with several properties of natural languages. For instance, the fact that dependency structures in natural languages are much less non-projective than in randomly ordered trees can be explained by DLM (Ferrer i Cancho, 2006; Liu, 2008). It is also claimed that DLM is a factor affecting the grammar of languages and word order choices (Gildea and Temperley, 2010; Temperley and Gildea, 2018).

Since the dependency length is equal to the dependency flux size, by trying to minimize the lengths of the dependencies, we also try to minimize the sizes of the inter-word fluxes. This gives us two different views on DLM. The objective of this article is to show that thinking about DLM in terms of flux has several advantages. In section 2, we will show that the interpretation of DLM in terms of flux makes it possible to highlight the cognitive relevance of this constraint. In section 3 , we will examine other fluxbased constraints related to DLM.

\section{Cognitive relevancy of DLM}

As we have just seen, DLM corresponds to the minimization of the flux size of the sentence and therefore of all inter-word fluxes. However, since we know that sentences are more or less parsed as fast as they are received by the speakers (Frazier and Fodor, 1978), we can see the flux in a given inter-word position as the information resulting from the portion of the sentence already analyzed that is necessary for its further analysis. In other words, there is an obvious link between the inter-word flux and the working memory of the recipient of an utterance (as well as the producer of the utterance).

The links between syntactic complexity and working memory have often been discussed, starting with Yngve (1960) and Chomsky and Miller (1963). According to Friederici (2011), "the processing of syntactically complex sentences requires some working memory capacity". The founding work on limitations of working memory is Miller's (1956), who defended that the span is $7 \pm 2$ elements; this limitation has been updated between 3 and 5 meaningful items by Cowan's work (2001). According to Cowan (2010), "Working memory is used in mental tasks, such as language comprehension (for example, retaining ideas from early in a sentence to be combined with ideas later on), problem solving (in arithmetic, carry a digit from the ones to the tens column while remembering the numbers), and planning (determining the best order in which to visit the bank, library, and grocery)." He adds that "There are also processes that can influence how effectively working memory is used. An important example is in the use of attention to fill working memory with items one should be remembering."

We think that the dependency flux in inter-word positions is a good approximation of what the recipient must remember to parse the rest of the sentence. Of course, it is also possible to make a link between the working memory and DLM if it is interpreted in terms of dependency length: it means that it is cognitively expensive to keep a dependency in working memory for a long time and that the longer a dependency is, the more likely it is to deteriorate in working memory (Gibson, 1998; 2000).

\section{DLM-related constraints}

DLM is a constraint on the size of the whole flux of a sentence and therefore a particular case of constraints on the complexity of the flux. DLM is neither the only metrics for syntactic complexity (see Lewis (1996) for several constituency-based metrics; Berdicevskis et al. 2018), nor the only metrics on the complexity of the flux and perhaps not the best. We will present other potentially interesting fluxbased metrics.

\subsection{Constraints on the size of inter-word fluxes}

We have seen that the sum of the lengths of the dependencies is equal to the sum of the sizes of the interword fluxes. Since there are as many dependencies as there are inter-word positions in a sentence $(n-1$ for a sentence of $n$ words), this means that the average length of the dependencies is equal to the average size of the inter-word fluxes. For the entire UD database (version 2.4, 146 treebanks), this value is equal 
to 2.73. But the equality of the average values does not mean that the values of these two variables, dependency length and flux size, are distributed in the same way. We give the two distributions in Figure 2.



Figure 2. Repartition of dependency lengths vs. flux sizes

For the distribution of dependencies according to their length, we observe that quantities decrease rapidly with lengths, starting with $47 \%$ of length 1 dependencies; for the distribution of inter-word fluxes according to their size, we observe a higher quantity of size 2 fluxes than size 1 ( $29 \%$ versus $23 \%)$, then a slower decrease at the beginning than for dependency lengths, then much faster. The two curves cross for the value 7. In fact, $99 \%$ of the fluxes are of size $\leq 7$, while for the dependency length, it is necessary to reach the value 17 to have more than $99 \%$ of dependencies of this length or less $(99.97 \%$ of fluxes of size $\leq 17$ vs. $99.09 \%$ of dependency lengths). Said differently, there are $0.91 \%$ of dependency lengths $\geq$ 18 against $0.03 \%$ of flux sizes, that is, about 30 times more (see Appendix 1 for more detailed results).

If we look at treebanks separately we see similar results: see table of Appendix 2 which shows the distribution values of 47 treebanks containing more than 100,000 flux positions. Looking at the curves of the percentages of dependency length and flux size, we notice the same crossing between values 1 and 2: The percentage of size 1 fluxes is always lower than the percentage of length 1 dependencies, while the percentage of size 2 fluxes is higher than the percentage of length 2 dependencies. Then, the percentage of fluxes decreases very quickly, and a second crossing is between the value 5 (UD_Finish-FTB), and the value 8 (in 9 treebanks: UD_Urdu-UDTB, UD_Persian-Seraji, UD_Hindi-HDTB, UD_German-HDT, UD German-GSD, UD Dutch-Alpino, UD Chinese-GSD, UD Arabic-PADT and UD Japanese-BC$\mathrm{CWJ})$. After this crossing, the percentage of flux size is lower than the percentage of dependency length and the former decreases much faster than the latter.

Looking at the cumulative percentages from value 2 onwards, the rate of flux size reduction is even sharper, as the crossing is between values 3 (in 4 treebanks: UD_Estonian-EDT, UD_Finnish-FTB, UD_Finnish-TDT, and UD_Polish-PDB) and 5 (in the same 9 treebanks as before). We notice in the treebanks with a crossing at 5 , most are the head-final languages like Japanese (UD_Japanese-BCCWJ), German (UD_German-GSD, UD_German-HDT), Dutch (UD_Dutch-Alpino) and Persian (UD_PersianSeraji), as well as Chinese (UD_Chinese-GSD) which are verb-initial position and head-final for other configurations. An exception is Arabic (UD_Arabic-PADT) which is a typical head-initial language. In treebanks with a crossing at 3, there are head-initial languages such as Finnish (UD_Finnish-FTB and UD_Finnish-TDT), Polish (UD_Polish-PDB) and Estonian (UD_Estonian-EDT). This could result from an asymmetry in the flux in languages according to the position of heads: For head-final languages, the flux size would be less constrained than for head-initial languages. This hypothesis remains to be confirmed by further study. 
If we look for which value $n$ we reach the $99 \%$ of dependencies of length $\leq n$, we find values ranging from 9 (UD_finish-FTB) to 27 (UD_Arabic-PADT). The same calculation for flux size gives values between 6 (in 12 treebanks: UD_Bulgarian-BTB, UD_Czech-FicTree, UD_Finnish-FTB, UD_FrenchGSD, UD_Italian-ISDT, UD_Korean-Kaist, UD_Norwegian-Bokmaal, UD_Polish-PDB, UD_Portuguese-GSD, UD_Romanian-RRT, UD_Russian-SynTagRus, and UD_Spanish-GSD) and 11 (UD_Japanese-BCCWJ) (this value is a little exceptional, since the value is equal to 7 for UD_Japanese-BC$\mathrm{CWJ})$. We also find that variations in dependency length are more sensitive than those in flux size in the different treebanks of a language. For example, for French, UD_French-FTB has $99 \%$ length dependencies $\leq 21$, and UD_French-GSD has $99 \%$ length dependencies $\leq 16$, while in the case of flux size, the values are 7 and 6 respectively.

If DLM expresses a constraint on the average value of dependency lengths and flux sizes, we see that there is also a fairly strong constraint on the size of each inter-word flux, whereas there is not such a strong constraint on the length of each dependency. For this reason, we postulate that DLM results more on a constraint on flux sizes than on dependency lengths, even if it is not possible to give a precise limit to the size of individual fluxes as Kahane et al. (2017) have already shown.

\subsection{Center-embedding and constraints on structured fluxes}

Beyond the question of their lengths, the way the dependencies are organized plays an important role in syntactic complexity. In particular, center-embedding structures carry a computational constraint in sentence processing (Chomsky and Miller, 1963; Lewis, 1996; Lewis and Vasishth, 2005). It is important to note that the complexity caused by center-embedding structures cannot be involved in DLM-based constraints. Neurobiological studies have highlighted the independence of memory degradation related to the length of a dependency and the computational aspect expressed by the center-embedding phenomena, which are located in different parts of the brain (Makuuchi et al., 2009).

As shown by Kahane et al. (2017), it is possible to express the constraints on the center-embedding in terms of constraints on the flux, but this requires to consider how all the dependencies belonging to the same flux are structured, by taking into account information about their vertices. Dependencies that share a vertex are referred to as a bouquet, while dependencies that have no common vertex are referred to as disjoint dependencies (Kahane et al., 2017). For example, the flux between climate and risks in Figure 1 contains 3 dependencies: the dependencies $<$ nmod and $>$ ccomp form a bouquet (they share the vertex risks), >ccomp and >advcl also (they share the vertex mitigate), while $<$ nmod and $>$ advcl are disjoint. The flux structure can be represented as shown by the table in Figure 3: vertices on the left of the considered inter-word position give the rows, beginning by the word which is closer to the position, while vertices on the right give the columns, beginning again by the word which is closer to the position. Dependencies which are on the same row or in the same column share a vertex.

\begin{tabular}{|l|l|l|}
\hline & risks & alleviating \\
\hline climate & $<\mathrm{nmod}$ & \\
\hline mitigate & $>\mathrm{ccomp}$ & $>\mathrm{advcl}$ \\
\hline
\end{tabular}

Figure 3. Structure of the flux in the position between climate and risks

The disjoint dependencies correspond to nested constructions. For example, in our example, the dependency between risks and climate and the dependency between mitigate and alleviating are disjoint and therefore the unit [risks,mitigate] is fully embedded in the unit [climate,alleviating]. ${ }^{2}$ The number of disjoint dependencies in a flux is very constrained as shown by Kahane et al. (2017): $99.62 \%$ of the fluxes in the UD database have less than 3 disjoint dependencies. This suggests that bouquet structures are less constrained than disjoint structures. This is quite predictable if we consider that there are con-

\footnotetext{
${ }^{2}$ In the case of a projective tree, the interval between two words connected by a dependency forms a connected portion of the structure and thus a syntactic unit.
} 
straints on working memory and that dependencies in a bouquet share more information than disjoint dependencies.

Note that non-projectivity can also be detected from a structured flux, if we take into account the order in which the vertices of the dependencies of the same flux are located. We plan in our further studies to look more precisely on the distribution of the different possible configurations of the flux.

\subsection{Constraints on the potential flux}

It must be remarked that we do not really know the flux when processing a sentence incrementally since we do not generally know which words already processed will be linked with a word not yet processed. We call potential flux in a given inter-word position the set of words before the position which are likely to be linked to words after it. See in particular the principles of the transition-based parsing (Nivre, 2003) which consists in keeping all the words already processed and still accessible in the working memory. The largest hypothesis on the potential flux is to consider that all words before the position are accessible. But clearly some words are more likely to have dependents (for instance, only content words can have dependents in UD). It is also possible to make structural hypothesis on the potential flux. We call projective potential flux the set of words accessible while maintaining the projectivity of the analysis. We will limit our study to the projective potential flux even if we are aware that, on one hand, projectivity is far to be an absolute constraint in many languages and, in the other hand, other constraints apply on the potential flux.

Figure 4 shows the value of the projective potential flux of our example. For instance, after while three words are accessible: mitigate, risks, and while; words before mitigate are not accessible because they are all depending on mitigate and climate is not accessible because it depends on risks and a projective link cannot cover an ancestor.



Figure 4. An ordered dependency tree with the projective potential flux of each inter-word position

Figure 5 compares the distribution of the sizes of potential projective fluxes and the sizes of observed fluxes for all UD treebanks (the sizes of observed fluxes have already been given in Figure 2).

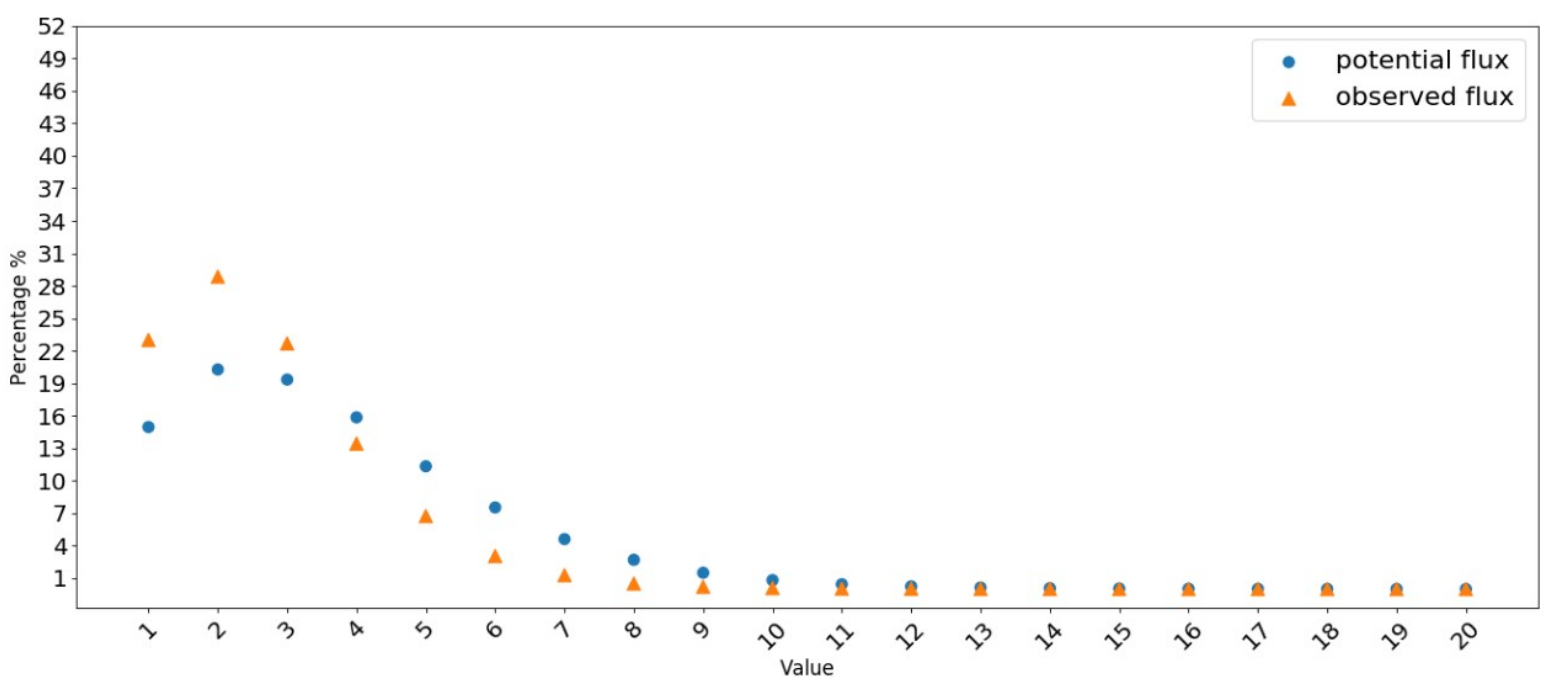

Figure 5. Distribution of projective potential fluxes vs observed fluxes 
The distribution of projective potential fluxes is flatter ( $20 \%$ vs. $29 \%$ for size 2 ) with less fluxes with sizes $\leq 3$ and more fluxes with sizes $\geq 4$, which means that projective potential fluxes generally have greater size than observed flux. From the size 2, the number of projective potential fluxes decreases but more slowly than the number of observed fluxes. It is necessary to reach size 11 to have more than $99 \%$ of the potential fluxes $(99.39 \%$ of the potential fluxes have a size $\leq 11)$ while this value is reached with size 7 for the observed fluxes (see Appendix 3 for details).

It is interesting to note that the projective potential flux is not the same for head-initial and head-final dependencies. If the governor is before its dependent, they are both accessible for further projective dependencies. But if the dependent is before the governor, only the governor is accessible for further projective dependencies. Consequently, we decided to compare the distribution of the sizes of the projective potential fluxes of two head-initial languages (Arabic and Irish) with those of two head-final languages (Japanese and German) (Figure 6). ${ }^{3}$



Figure 6. Comparison of projective potential flux sizes for head-initial and head-final languages

For head-final languages, the distribution of projective potential flux sizes is similar to the general distribution presented in Figure 5: Projective potential fluxes with size 2 are the most numerous, 22\% for Japanese and $20.5 \%$ for German. Then the percentage reduces with the increase in size, the two finalheaded languages have a percentage fairly close for size 7 . More than $99 \%$ of projective potential fluxes have a size $\leq 10$ for Japanese and $\leq 11$ for German.

As expected, the distribution of projective potential flux sizes is different for head-initial languages: Projective potential fluxes with size 3 (Irish) and size 4 (Arabic) are the most numerous (21\% for Irish and $15 \%$ for Arabic). Compared to head-final languages, not only does the percentage of projective potential flux size in head-initial languages increase more slowly (than for head-final languages) to reach the most represented size, but also it decreases more slowly afterwards. Thus, the distribution of headinitial languages is flatter than for head-final language and Arabic is particularly flat, which means that there are much more projective potential fluxes with greater sizes. From size 8 onwards, the distribution of Irish is very close to that of the two head-final languages. But in the case of Arabic, the distribution only approaches the other three from size 15 . If we look at the cumulative percentage, more than $99 \%$ of projective potential flux have a size $\leq 11$ for Irish and $\leq 15$ for Arabic.

This difference in the distribution of projective potential fluxes for head-initial and head-final languages could have some consequences. Figure 7 shows the observed flux sizes for the same four languages.

\footnotetext{
${ }^{3}$ German is V2 (verb second position) in main clauses and head-final in subordinated clauses.
} 


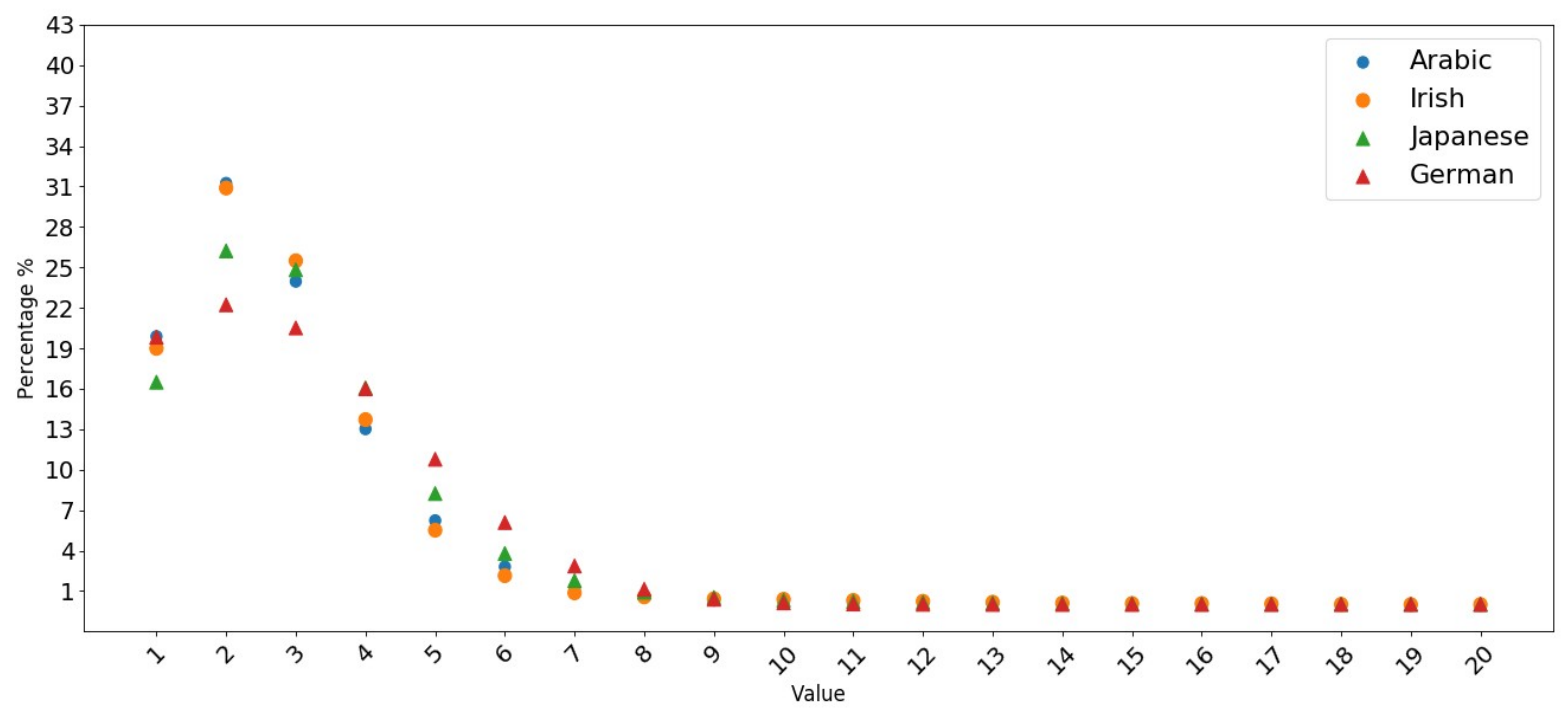

Figure 7. Comparison of observed flux sizes for head-initial and head-final languages

We have already noted that in UD treebanks, projective potential fluxes tend to have larger sizes than those of observed fluxes (Figure 5), but this trend is accentuated in head-initial languages: Projective potential fluxes with small sizes are much less numerous than for observed fluxes: For Arabic, $5 \%, 9 \%$, and $13 \%$ of projective potential fluxes with sizes 1,2 , and 3 compared to $20 \%, 31 \%, 24 \%$ of observed fluxes. For Irish, $8 \%, 17 \%$ and $21 \%$ of projective potential fluxes with sizes 1,2 , and 3 compared to $19 \%, 31 \%$, $25.5 \%$ of observed fluxes. Consequently, $73 \%$ of the potential fluxes with a size $\geq 4$ compared to $25 \%$ of the observed fluxes for Arabic, and 54\% compared to 25\% for Irish.

It could seem contradictory that the projective potential flux is larger in head-initial languages (than in head-final languages), while the observed flux is significantly smaller (we have more fluxes with small sizes). It may be the result of a phenomenon of compensation: A larger potential flux increases the complexity of the (human, as well as automatic) parsing and a smaller observed flux would compensate for this. We do not have a better explanation for the time being and we leave this question open for further studies based on a deeper analysis of the data.

\section{Conclusion}

We have shown that dependency length minimization (DLM) is also a property of inter-word dependency fluxes. Such a view allows us to reformulate many assumptions on DLM. For instance, Gildea and Temperley (2018) remark that the idea that languages tend to place closely related words close together can be expressed as DLM. But as DLM comes down to reduce the flux and therefore the working memory, this can be reformulated by saying that the idea that languages tend to place closely related words close together can be expressed as a reduction of working memory.

We hope that this article will motivate studies on constraints on dependency flux, which are not limited to DLM. In particular, we believe that the constraints on the flux are far to be limited to its average size and that the structure of the flux plays an important role in its complexity. We have in particular shown an asymmetry between head-initial and head-final languages concerning the flux that could be related to the different potential flux in these two kind of languages.

\section{Acknowledgments}

We would like to thank our three reviewers for valuable remarks. 


\section{References}

Berdicevskis Aleksandrs et al. 2018. Using Universal Dependencies in cross-linguistic complexity research. In Proceedings of the Second Workshop on Universal Dependencies (UDW 2018), 8-17.

Ramon Ferrer i Cancho. 2006. Why do syntactic links not cross?. EPL (Europhysics Letters), 76, 1228-1234.

Nelson Cowan. 2001. The magical number 4 in short-term memory: A reconsideration of mental storage capacity. Behavioral and brain sciences, 24(1), 87-114.

Nelson Cowan. 2010. The magical mystery four: How is working memory capacity limited, and why?. Current directions in psychological science, 19(1), 51-57.

Angela D Friederici. 2011. The brain basis of language processing: from structure to function. Physiological reviews, 91(4), 1357-1392.

Lyn Frazier and Fodor Janet Dean. 1978. The sausage machine: A new two-stage parsing model, Cognition, 6(4), 291-325.

Richard Futrell, Kyle Mahowald and Edward Gibson. 2015. Large-scale evidence of dependency length minimization in 37 languages. Proceedings of the National Academy of Sciences, 112(33), 10336-10341.

Edward Gibson. 1998. Linguistic complexity: Locality of syntactic dependencies. Cognition, 68(1), 1-76.

Edward Gibson. 2000. The dependency locality theory: A distance-based theory of linguistic complexity. Image, language, brain, 2000, 95-126.

Daniel Gildea and David Temperley. 2010. Do grammars minimize dependency length?. Cognitive Science, 34(2), 286-310.

Sylvain Kahane, Alexis Nasr and Owen Rambow. 1998. Pseudo-projectivity: a polynomially parsable non-projective dependency grammar. In COLING 1998 Volume 1: The 17th International Conference on Computational Linguistics (Vol. 1).

Sylvain Kahane, Chunxiao Yan, and Marie-Amélie Botalla. 2017. What are the limitations on the flux of syntactic dependencies? Evidence from UD treebanks. In 4th international conference on Dependency Linguistics (Depling) (pp. 73-82).

Richard L. Lewis. 1996. Interference in short-term memory: The magical number two (or three) in sentence processing. Journal of psycholinguistic research, 25(1), 93-115.

Richard L. Lewis and Shravan Vasishth. 2005. An activation-based model of sentence processing as skilled memory retrieval. Cognitive science, 29(3), 375-419.

Haitao Liu. 2008. Dependency distance as a metric of language comprehension difficulty. Journal of Cognitive Science, 9(2), 159-191.

Michiru Makuuchi et al. 2009. Segregating the core computational faculty of human language from working memory. Proceedings of the National Academy of Sciences, 106(20), 8362-8367.

George A. Miller and Noam Chomsky. 1963. Finitary models of language users.

Joakim Nivre. 2003. An efficient algorithm for projective dependency parsing. In Proceedings of the Eighth International Conference on Parsing Technologies, 149-160.

Joakim Nivre. 2006. Constraints on non-projective dependency parsing. In 11th Conference of the European Chapter of the Association for Computational Linguistics.

Daniel D. K. Sleator and Davy Temperley. 1995. Parsing English with a link grammar. arXiv preprint cmp-lg/ 9508004.

David Temperley and Daniel Gildea. 2018. Minimizing syntactic dependency lengths: typological/cognitive universal?. Annual Review of Linguistics, 4, 67-80.

Victor H. Yngve. 1960. A model and an hypothesis for language structure. Proceedings of the American philosophical society, 104(5), 444-466. 
Appendix 1. Results of distribution of dependency lengths and flux sizes $\leq \mathbf{2 0}$

\begin{tabular}{|c|c|c|c|c|}
\hline $\mathrm{n}$ & $\% \mathrm{DL}=\mathrm{n}$ & $\% \mathrm{DL} \leq \mathrm{n}$ & $\% \mathrm{FS}=\mathrm{n}$ & $\% \mathrm{FS} \leq \mathrm{n}$ \\
\hline 0 & 0 & 0 & 0 & 0 \\
\hline 1 & 47.26 & 47.26 & 23 & 23 \\
\hline 2 & 22.66 & 69.92 & 28.84 & 51.84 \\
\hline 3 & 10.59 & 80.51 & 22.68 & 74.51 \\
\hline 4 & 5.52 & 86.03 & 13.43 & 87.94 \\
\hline 5 & 3.46 & 89.49 & 6.74 & 94.69 \\
\hline 6 & 2.38 & 91.87 & 3.05 & 97.74 \\
\hline 7 & 1.72 & 93.59 & 1.28 & 99.02 \\
\hline 8 & 1.3 & 94.89 & 0.51 & 99.53 \\
\hline 9 & 1 & 95.89 & 0.21 & 99.74 \\
\hline 10 & 0.78 & 96.66 & 0.09 & 99.83 \\
\hline 11 & 0.61 & 97.28 & 0.05 & 99.88 \\
\hline 12 & 0.49 & 97.76 & 0.03 & 99.91 \\
\hline 13 & 0.39 & 98.15 & 0.02 & 99.93 \\
\hline 14 & 0.31 & 98.46 & 0.01 & 99.95 \\
\hline 15 & 0.25 & 98.71 & 0.01 & 99.96 \\
\hline 16 & 0.21 & 98.92 & 0.01 & 99.96 \\
\hline 17 & 0.17 & 99.09 & 0.01 & 99.97 \\
\hline 18 & 0.14 & 99.22 & 0 & 99.97 \\
\hline 19 & 0.11 & 99.34 & 0 & 99.98 \\
\hline 20 & 0.09 & 99.43 & 0 & 99.98 \\
\hline
\end{tabular}


Appendix 2. Results of distrubution of dependency lengths (DLs) and flux sizes (FSs) in 47 treebanks

\begin{tabular}{|c|c|c|c|c|c|}
\hline & $99 \%$ DLs $\leq \mathrm{n}$ & $99 \%$ Fss $\leq \mathrm{n}$ & $\begin{array}{l}\text { Crossing value } \\
\% \mathrm{DL}>\% \mathrm{FS}\end{array}$ & $\begin{array}{l}\text { Crossing value } \\
\% \mathrm{FS}>\% \mathrm{DL}\end{array}$ & $\begin{array}{l}\text { Crossing value cu- } \\
\text { mul } \% \text { FS }>\text { cumul } \\
\% \text { DL }\end{array}$ \\
\hline UD_Ancient_Greek-PROIEL & 17 & 8 & 7 & 2 & 4 \\
\hline UD_Ancient_Greek-Perseus & 16 & 8 & $7 *$ & $2^{*}$ & 4 \\
\hline UD_Arabic-NYUAD & 24 & 9 & 7 & 2 & 4 \\
\hline UD_Arabic-PADT & 27 & 9 & 8 & 2 & 5 \\
\hline UD_Bulgarian-BTB & 12 & 6 & 6 & 2 & 4 \\
\hline UD_Catalan-AnCora & 19 & 7 & 7 & 2 & 4 \\
\hline UD_Chinese-GSD & 22 & 9 & 8 & 2 & 5 \\
\hline UD_Croatian-SET & 15 & 7 & 7 & 2 & 4 \\
\hline UD_Czech-CAC & 15 & 7 & 6 & 2 & 4 \\
\hline UD_Czech-FicTree & 12 & 6 & 6 & 2 & 4 \\
\hline UD_Czech-PDT & 14 & 7 & 6 & $2 *$ & 4 \\
\hline UD_Dutch-Alpino & 17 & 8 & 8 & 2 & 5 \\
\hline UD_English-EWT & 15 & 7 & 7 & 2 & 4 \\
\hline UD_Estonian-EDT & 12 & 7 & 6 & 2 & 3 \\
\hline UD_Finnish-FTB & 9 & 6 & 5 & 2 & 3 \\
\hline UD_Finnish-TDT & 12 & 7 & 6 & 2 & 3 \\
\hline UD_French-FTB & 21 & 7 & 7 & 2 & 4 \\
\hline UD_French-GSD & 16 & 6 & 6 & 2 & 4 \\
\hline UD_Galician-CTG & 17 & 7 & 7 & 2 & 4 \\
\hline UD_German-GSD & 17 & 8 & 8 & 2 & 5 \\
\hline UD_German-HDT & 17 & 8 & 8 & 2 & 5 \\
\hline UD_Hebrew-HTB & 18 & 7 & 6 & 2 & 4 \\
\hline UD_Hindi-HDTB & 21 & 8 & 8 & 2 & 5 \\
\hline UD_Italian-ISDT & 16 & 6 & 6 & 2 & 4 \\
\hline UD_Italian-PoSTWITA & 17 & 7 & 7 & 2 & 4 \\
\hline UD_Italian-VIT & 19 & 7 & 7 & 2 & 4 \\
\hline UD_Japanese-BCCWJ & 26 & 11 & 8 & 2 & 5 \\
\hline UD_Japanese-GSD & 21 & 7 & 7 & 2 & 4 \\
\hline UD_Korean-Kaist & 14 & 6 & 6 & 2 & 4 \\
\hline UD_Latin-ITTB & 15 & 7 & 6 & 2 & 4 \\
\hline UD_Latin-PROIEL & 17 & 8 & 7 & 2 & 4 \\
\hline UD_Latvian-LVTB & 13 & 7 & 6 & 2 & 4 \\
\hline
\end{tabular}




\begin{tabular}{|l|l|l|l|l|l|}
\hline UD_Norwegian-Bokmaal & 13 & 6 & 6 & 2 & 4 \\
\hline UD_Norwegian-Nynorsk & 14 & 7 & 6 & 2 & 4 \\
\hline UD_Old_French-SRCMF & 12 & 7 & 6 & 2 & 4 \\
\hline UD_Old_Russian-TOROT & 14 & 8 & 6 & 2 & 4 \\
\hline UD_Persian-Seraji & 24 & 9 & 8 & 2 & 5 \\
\hline UD_Polish-PDB & 13 & 6 & 6 & 2 & 3 \\
\hline UD_Portuguese-Bosque & 17 & 7 & 7 & 2 & 4 \\
\hline UD_Portuguese-GSD & 17 & 6 & 6 & 2 & 4 \\
\hline UD_Romanian-Nonstandard & 15 & 7 & 6 & 2 & 4 \\
\hline UD_Romanian-RRT & 15 & 6 & 6 & 2 & 4 \\
\hline UD_Russian-SynTagRus & 14 & 6 & 6 & 2 & 4 \\
\hline UD_Slovenian-SSJ & 14 & 7 & 7 & 2 & 4 \\
\hline UD_Spanish-AnCora & 18 & 7 & 7 & 2 & 4 \\
\hline UD_Spanish-GSD & 17 & 6 & 9 & 6 & 5 \\
\hline UD_Urdu-UDTB & 24 & 6 & 6 & 2 \\
\hline
\end{tabular}

* There are several crosses, but they are after the value 40 which are rather unrepresentative. 
Appendix 3. Results of distribution of projective potential flux $\leq \mathbf{2 0}$

\begin{tabular}{|c|c|c|}
\hline $\mathrm{n}$ & $\%$ Projective potential flux $=\mathrm{n}$ & $\%$ Projective potential flux $\leq \mathrm{n}$ \\
\hline 0 & 0 & 0 \\
\hline 1 & 14.96 & 14.96 \\
\hline 2 & 20.27 & 35.23 \\
\hline 3 & 19.34 & 54.57 \\
\hline 4 & 15.85 & 70.42 \\
\hline 5 & 11.34 & 81.76 \\
\hline 6 & 7.53 & 89.29 \\
\hline 7 & 4.61 & 93.9 \\
\hline 8 & 2.7 & 96.6 \\
\hline 9 & 1.51 & 98.11 \\
\hline 10 & 0.83 & 98.94 \\
\hline 11 & 0.45 & 99.39 \\
\hline 12 & 0.25 & 99.64 \\
\hline 13 & 0.14 & 99.78 \\
\hline 14 & 0.08 & 99.86 \\
\hline 15 & 0.05 & 99.91 \\
\hline 16 & 0.03 & 99.94 \\
\hline 17 & 0.02 & 99.96 \\
\hline 18 & 0.01 & 99.97 \\
\hline 19 & 0.01 & 99.98 \\
\hline 20 & 0.01 & 99.99 \\
\hline
\end{tabular}

